\title{
A NOTE ON THE IDENTIFICATION OF LINEAR SYSTEMS ${ }^{1}$
}

\author{
RICHARD BELLMAN
}

1. Introduction. Consider a linear system described by the function $u(t)$ satisfying the linear differential equation

$$
u^{(N)}+a_{1}(t) u^{(N-1)}+\cdots+a_{N}(t) u=0,
$$

where the $a_{i}(t)$ are continuous functions of $t$ in an interval [0,T]. The initial conditions, $u^{(i)}(0)=c_{i}, i=0,1, \cdots, N-1$, then determine the function $u(t)$ uniquely. The situation is, however, much more complicated, and therefore interesting, if we allow multipoint conditions of the type

$$
u\left(t_{i}\right)=c_{i}, \quad i=1,2, \cdots, N,
$$

where $0 \leqq t_{1} \leqq t_{2} \leqq \cdots \leqq t_{N}$. Coincidence of the $t_{i}$ corresponds to conditions on the derivatives of $u(t)$. A very elegant result due to Polya [1] (see also Beckenbach and Bellman [2] for further discussion and references) resolves the problem of determining when (1.2) determines $u(t)$ for an arbitrary set of $t$-points.

From the standpoint of identification theory, we are asking that observation of the system at any $N$ isolated times in the interval $[0, T]$ allow us to prescribe the behavior over the entire interval. There are many ways of extending the scope of this problem. We can consider more general systems described by more general functional equations, such as differential-difference equations, partial differential equations, or nonlinear equations, or we can treat more general types of "observations."

We shall pursue the latter path here. In place of (1.2), consider the $N$ conditions

$$
\int_{0}^{T} u(t) \phi_{j}(t) d t=b_{j}, \quad j=1,2, \cdots, N,
$$

where the $\phi_{j}$ are given functions. We shall show that the solution of (1.1) is determined in this fashion, provided we impose certain conditions on $u(t)$ and the $\phi_{j}$. These conditions are closely allied to the condition imposed by Polya, condition $W$.

Received by the editors December 23, 1964.

1 This research was carried out while at the RAND Corporation in Santa Monica. 
2. Preliminaries. Let $\left\{u_{j}(t)\right\}, j=1,2, \cdots, N$, be $N$ linearly independent solutions of (1.1). Write the general solution in the form

$$
u=\sum_{i=1}^{N} c_{i} u_{i}(t)
$$

Then the integral conditions of (1.3) reduce to the system of linear algebraic equations

$$
\sum_{i=1}^{N} c_{i} \int_{0}^{T} u_{i}(t) \phi_{j}(t) d t=b_{j}, \quad j=1,2, \cdots, N .
$$

The determination of a unique function $u(t)$ satisfying both (1.1) and (1.3) has thus been converted into an examination of the nonvanishing of the determinant

$$
\left|\int_{0}^{T} u_{i}(t) \phi_{j}(t) d t\right|, \quad i, j=1,2, \cdots, N .
$$

To study this question we invoke an extremely useful determinantal identity

$$
\begin{aligned}
&\left|\int_{0}^{T} u_{i}(t) \phi_{j}(t) d t\right| \\
&(2.4)=\frac{1}{N !} \int_{0}^{T} \cdots \int_{0}^{T} P\left(t_{1}, t_{2}, \cdots, t_{N}, u\right) \\
& \cdot P\left(t_{1}, t_{2}, \cdots, t_{N}, \phi\right) d t_{1} d t_{2} \cdots d t_{N},
\end{aligned}
$$

where

$$
P\left(t_{1}, t_{2}, \cdots, t_{N}, u\right)=\left|\begin{array}{llll}
u_{1}\left(t_{1}\right) & u_{1}\left(t_{2}\right) & \cdots & u_{1}\left(t_{N}\right) \\
u_{2}\left(t_{1}\right) & u_{2}\left(t_{2}\right) & \cdots & u_{2}\left(t_{N}\right) \\
\vdots & & \\
u_{N}\left(t_{1}\right) & u_{N}\left(t_{2}\right) & \cdots & u_{N}\left(t_{N}\right)
\end{array}\right|
$$

the Polyan of the $N$ functions $u_{1}(t), u_{2}(t), \cdots, u_{N}(t)$.

In the case $N=2$, the symmetry of the integrand in (2.4) about the line $t_{1}=t_{2}$ permits us readily to conclude that

$$
\left|\int_{0}^{T} u_{i}(t) \phi_{j}(t) d t\right|=\iint_{0 \leq t_{1} \leq t_{2} \leq T} P\left(t_{1}, t_{2}, u\right) P\left(t_{1}, t_{2}, \phi\right) d t_{1} d t_{2} .
$$

In general, we can conclude that 


$$
\begin{aligned}
&\left|\int_{0}^{T} u_{i}(t) \phi_{j}(t) d t\right|=\int_{0 \leq t_{1} \leq t_{2} \leq} \cdots \int_{\substack{t_{N} \leq T\\
}} P\left(t_{1}, \cdots, t_{N}, u\right) \\
& P\left(t_{1}, \cdots, t_{N}, \phi\right) d t_{1} d t_{2} \cdots d t_{N} .
\end{aligned}
$$

This relation is the key to what follows.

3. Čebyšev systems. Let $\left\{u_{i}(t)\right\}, i=1,2, \cdots, N$, be a set of $N$ linearly independent continuous functions. We say that it is a

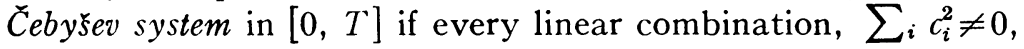

$$
f(t)=\sum_{i=1}^{N} c_{i} u_{i}(t)
$$

has at most $N$ zeros in $[0, T]$. A basic result (see Gantmacher-Krein $[3]$ ) is that a necessary and sufficient condition for $\left\{u_{i}\right\}$ to be a Cebyšev system is that

$$
P\left(t_{1}, t_{2}, \cdots, t_{N}, u\right) \neq 0
$$

for any set of $t_{i}$ in $[0, T]$ satisfying the condition

$$
0 \leqq t_{1}<t_{2}<\cdots<t_{N} \leqq T .
$$

It follows that $P$ has the same sign in this region.

Examples of Cebyšev systems are $\left\{t^{i}\right\}, i=0,1, \cdots, N-1$,

$$
\left\{\exp \left(\lambda_{i} t\right)\right\}, \lambda_{1}<\lambda_{2}<\lambda_{3}<\cdots<\lambda_{N} .
$$

Sometimes the oscillation condition is easy to verify; sometimes the determinantal condition.

4. A sufficient condition for identification. Using the foregoing results, we can assert:

Theorem. If any particular set of $N$ linearly independent solutions of (1.1) is a C Cebyšev set in [0,T] (and hence every such set), and if the $\phi_{j}$ are a Cebysev set, then the conditions of (1.3) determine u( $(t)$ uniquely.

It is clear that this condition on the solutions of $L(u)=0$ is a characteristic value condition. For example,

$$
u^{\prime \prime}+u=0
$$

satisfies the condition in $[0, T]$ if $T<2 \pi$, but not for $T \geqq 2 \pi$, as $u=\sin t$ shows. In general, we can assert that $L(u)=0$ satisfies the condition if $T$ is sufficiently small. 


\section{REFERENCES}

1. G. Polya, On the mean-value theorem corresponding to a given linear homogeneous differential equation, Trans. Amer. Math. Soc. 24 (1922), 312-324.

2. E. F. Beckenbach and R. Bellman, Inequalities, Springer, Berlin, 1961.

3. F. R. Gantmacher and M. G. Krein, Oszillationsmatrizen, Oszillationskerne und kleine Schwingungen mechanischer Systeme, Akademie-Verlag, Berlin, 1960.

RAND Corporation, Santa Monica

\section{A VOLTERRA EQUATION WITH A VERY SINGULAR KERNEL ${ }^{1}$}

E. L. ROETMAN

For continuous, or mildly singular kernels, the Volterra equations of second kind can be solved with a Neumann series which converges for the entire complex $\lambda$ plane. The purpose of the present note is to show, by studying a special case, what can happen if the kernel is very singular. In particular, we find that the equation can have finite spectral values, in fact the circle $|\lambda|=1$ can be a natural boundary for the resolvent kernel, and that it is not easy to describe a "natural" domain and range for the operator in terms of the usual classes of functions.

Consider the Volterra type integral equation for complex valued functions of a real variable given by

$$
\phi=a+\lambda k * \phi
$$

where

$$
k(t)=\left(i / \pi^{1 / 2}\right) t^{-3 / 2} \exp (i \pi / 4+i / t)
$$

and * denotes the convolution,

$$
f * g=\int_{\theta}^{t} f(t-s) g(s) d s .
$$

(The study of the boundary value problem for the equation of vibration of elastic bars leads to the related equation

Received by the editors November 13, 1964.

${ }^{1}$ Based in part on the author's thesis, written under the guidance of Professor W. Fulks, submitted to Oregon State University. 